\title{
Tour Planning Mechanism Using CLR Method to Achieve Reliability Control in WSN
}

\author{
Shraddha Patil ${ }^{1}$, Madhav Ingle ${ }^{2}$ \\ ${ }^{1}$ Savitribai Phule Pune University, Department of Computer Engineering, JSPM's JSCOE, Hadapsar, Pune-28, India \\ ${ }^{2}$ Professor, Department of Computer Engineering, JSPM's JSCOE, Savitribai Phule Pune University, Hadapsar, Pune-28, India
}

\begin{abstract}
In this proposed research, by introducing mobility into the network, a new data-gathering mechanism for large-scale wireless sensor networks is proposed. An M-collector - a mobile data collector could be a mobile robot or vehicle equipped with a powerful transceiver and battery. It works like a mobile base station and gathers data while moving in the field. An M-collector, periodically gathers the data from the static data sink. Then it polls data from each sensor node when it traverse through its transmission range. Later MDCN collects the data from the sensor node directly in a single-hope communications and finally transports all the collected data to static sink node. The lifetime of sensor is expected to be prolonged as the data packets are directly gathered without relays and collisions. In this research methodology, the main focus is to minimize the length of each data gathering tour which is referred as SHDGP which is single-hop data gathering problem.
\end{abstract}

Keywords: MDCN, WSN, SHDGP

\section{Introduction}

A new data-gathering mechanism is introduced in large scale wireless sensor network. M-collector which means a mobile data collector could be a robot. It is nothing but a vehicle equipped having powerful transceiver and battery which work like a mobile base station and gathers the data when it moves through the field. First an M-collector node starts the tour to gather data from other sensor nodes in a single-hop communications. And finally it transports the data the sink node.

Sensor nodes are deployed in remote environments. Those are deployed in multi-hop WSN over wide range of area. In cluster based architecture, all sensor nodes sense the data and transfer it to sink node. Nodes which are closer to sink node gets overloaded and due to which their lifetime is decreased. So the main disadvantage of this method is lifetime of sensor node is decreased also energy is lost. To overcome this problem, we propose new mechanism with the help of tour planning using MDCN in the network in which MDCN alone transfers the data to sink node. When buffer gets full, new incoming data can be lost. To avoid this, we increase reliability by attaching database at each sensor node.

When the threshold value of buffer is reached, new incoming data can be discarded. And the data can be lost. There were no mechanisms present before to overcome this problem. In this proposed model, we achieve two main factors 1. Energy Efficient and 2. Reliability Control. The rest of this paper is organized as follows: Section 2 presents literature review about WSN and reliability control, Section 3 describes the proposed model. Section 4 shows the implementation results and analysis. Finally, the conclusion is presented in Section 5 .

\section{Literature Review}

First, a survey on efficient data collection in wsn is done. The TDMA (Time Division Multiple Access) concept is used to efficiently collecting sensor data for any traffic patterns. Thereby reducing the latency of data collections. Urgency of the data is not considered [1].

Data aggregation in wireless sensor network proposes data aggregation methods to gather and aggregate data in an energy efficient manner so that network lifetime is enhanced [2].

An Improving network efficiency by removing energy holes in wireless sensor network proposes energy efficient hole removing mechanism (EFORM) technique which is used to remove energy holes. Sleep and awake techniques are used and which requires an energy [3].

A review on data collection method with sink node in wireless sensor network mainly focuses on data collection method which make communication feasible between sink node and sensor node. MULE technique is used and because of which lots of energy is consumed [4].

Optimum packet length over data transmission for wireless sensor networks proposes optimum packet length over data transmission at the data link layer is decided. But packet length decision still remains the problem [5].

Energy balanced routing method for in-network data aggregation in wireless sensor networks proposes enhanced forward aware factor energy balanced routing method (EFAF-EBRM) based on data aggregation technique is used [6]. An efficient deduction of data loss rate in wireless sensor loss rate in wireless sensor network proposes the shortest path between the sensor nodes have been found and also data aggregation at the sensor nodes are performed so as to avoid bogus messages [7]. 


\section{International Journal of Science and Research (IJSR) \\ ISSN (Online): 2319-7064}

Index Copernicus Value (2015): 78.96 | Impact Factor (2015): 6.391

\section{Proposed Model}

The proposed model consists of three main things. 1. Sensor node 2. Cluster Node and 3. MDCN. All three is having their main functionalities as shown in the figure.

\subsection{System Architecture}

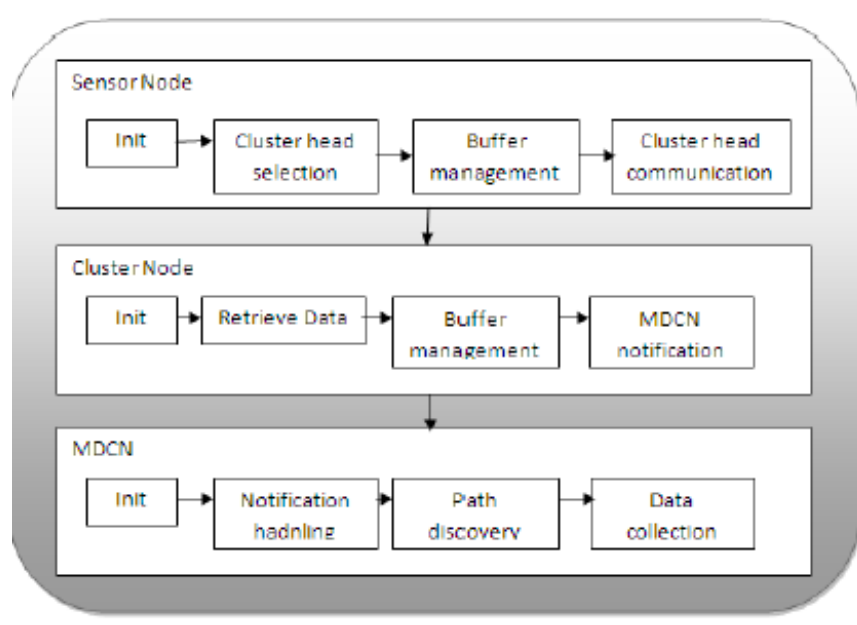

Figure 1: Proposed System Design

All the three modules and their functionalities is explained below.

3.1.1 Sensor Node: All sensor nodes present in WSN are initialized. Later the CSA (Cluster Selection Algorithm) is used and cluster head is selected. Every sensor node has its own buffer whose management can be done. All sensor nodes present in a cluster, sense data and periodically sends it to cluster head.

3.1.2 Cluster Node: In this module all cluster nodes are initialized. Data from sensor nodes is being retrieved periodically by the cluster node. Like sensor nodes, cluster node also has its own buffer attached. As soon as the buffer attached to cluster node gets full, the notification is sent to MDCN to collect data from it. As MDCN gets notification, it moves and collects the data from cluster node.

3.1.3 MDCN: At very first MDCN is initialized. The number of MDCNs used in the network can be decided on the area of wireless sensor network. If its large then one MDCN cannot be sufficient.

\section{Performance Evaluation}

We verify our analysis results and evaluate performance of proposed Tired Structure Data Aggregation Model. Here we consider

\subsection{Experimental Setup}

Table 1 shows an information about the experimental setup used and its result analysis.
Table 1: Experimental Setup

\begin{tabular}{|c|c|}
\hline Parameter & Value \\
\hline Area of Sensor Field & $500 * 500$ \\
\hline Topology & Mesh \\
\hline No. of Nodes & 50 \\
\hline No. of Sink Node & 1 \\
\hline Circular Layers & 7 \\
\hline Packet Size & Variable \\
\hline MAC Layer Protocol & $802.11(\mathrm{~b})$ \\
\hline Routing Protocol & AODV \\
\hline $\begin{array}{c}\text { Frequency of Packet Generation } \\
(1 / \text { Time Interval) : Reporting Rate }\end{array}$ & $\begin{array}{c}\text { Used different frequency } \\
\text { rate for analysis }\end{array}$ \\
\hline Source Nodes & $\begin{array}{c}\text { All common sensor nodes } \\
\text { within network }\end{array}$ \\
\hline
\end{tabular}

\subsection{Reliability Control Parameters}

We will calculate reliability control of wireless sensor network using tour planning mechanism, on following parameters-

\section{a) Packet Delivery Ratio}

Fig. 2 shows the NS3 generated graph for packet delivery ratio. Here packet size is 64 bytes and from the graph it can be seen that as the time increases packet delivery also increases. For first few seconds the increase rate is slow and it is suddenly increased later and remains stable thereafter.

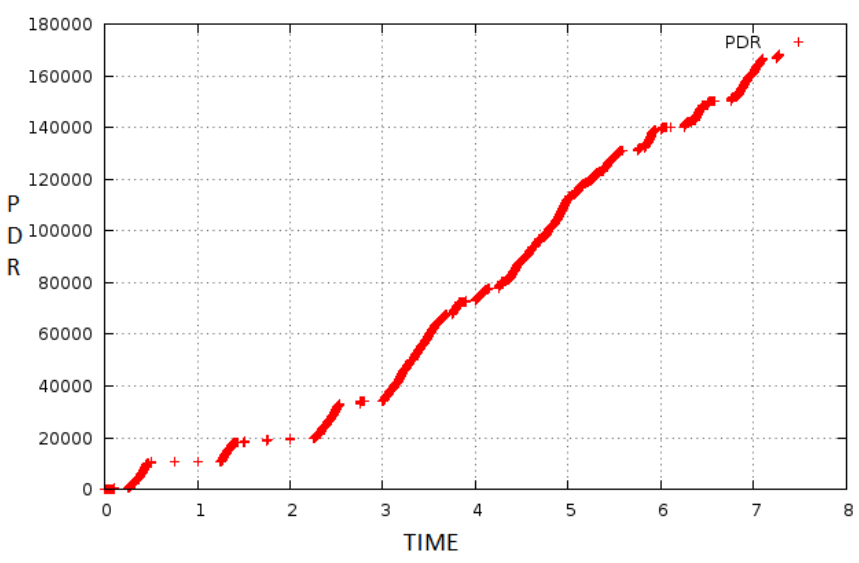

Figure 2: Packet Delivery Ratio

\section{b) Packet Count Ratio}

Fig 3 shows the NS3 generated results for packet count ratio for packet size 64 bytes. From the graph it is clear that with simulation time packet count also increases to a great extent which automatically results in decrease in packet loss.

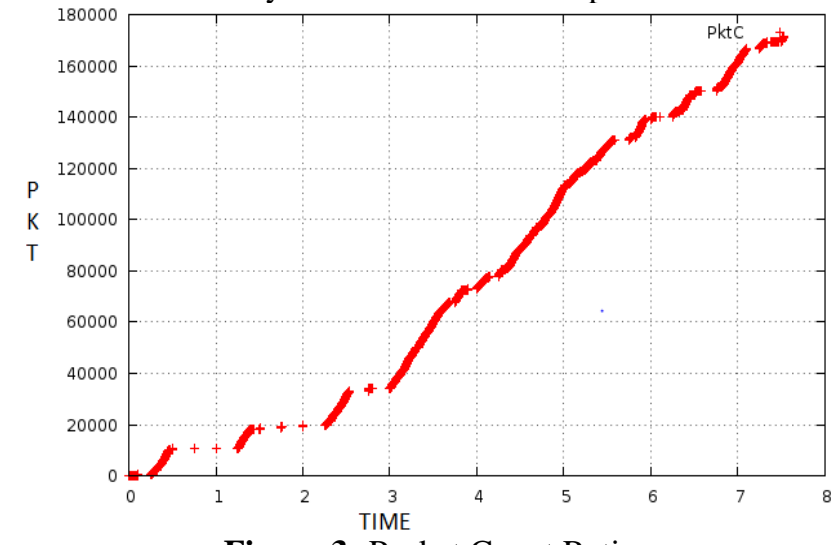

Figure 3: Packet Count Ratio 


\section{International Journal of Science and Research (IJSR) \\ ISSN (Online): 2319-7064}

Index Copernicus Value (2015): 78.96 | Impact Factor (2015): 6.391

c) Throughput

Fig 4 shows the NS3 generated results for Throughput of the network for packet size 64 bytes. From the graph it is clear that with simulation time throughput of the network also increases to a great extent due to less packet drop and E2E delay.

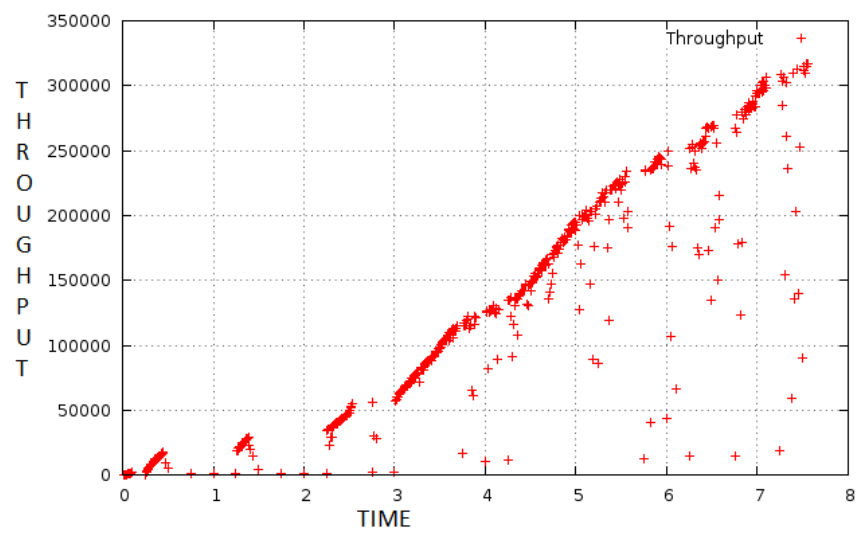

Figure 2: Throughput

\section{Conclusion}

In this paper, a new mobile data gathering scheme is proposed for large scale sensor networks. A mobile data collector also called an M-collector is been introduced. It works like a mobile base station in the network. An Mcollector node moves into the wireless sensor network and gathers data from all cluster heads and transfers it to the sink node. This proposed mechanism solves the problem of large scale homogeneous networks and also improves scalability. Because of M-collector concept, data gathering problem is resolved in unexpected topology of network. The simulation results demonstrates that our data gathering scheme reduces the moving length greatly.

In future security of the network on which the work can be carried. Also if the disaster happens unexpectedly, then the data can be lost greatly. So work can be carried on these two main points.

\section{References}

[1] R. Yogeswari, V.Subathra "A Survey on efficient data collection in WSN" in An ISO3297: 2007 Certified OrganizationVol. 1, Issue 9, November 2013.

[2] S.Wankhede, N.Chavan, "A Review on data collection method with sink node in WSN", in International Journal of Distributed and Parallel Systems (IJDPS) Vol.4, No.1, January 2013.

[3] S. Nandini, Prof. P. R. Patil, "Data Aggregation in Wireless Sensor Network", in IEEE International Conference on Computational Intelligence and Computing Research.

[4] M. B. Rasheed, N. Javaid, M. A. Khan, S. H. Bouk, "Improving Network Efficiency by Removing Energy Holes in WSN", in IEEE Communications Surveys Tutorials, Vol. 14, No. 2, Second Quarter 2012.

[5] j.K. Baby, P K Poonguzhali, "Energy Balanced Routing Method for In-Network Data Aggregation in Wireless
Sensor Networks", in IOSR Journal of Electronics and Communication Engineering (IOSR-JECE).

[6] M.Dener,"Optimum Packet Length Over Data Transmission for Wireless Sensor Networks", in proceedings of the 8th International Conference on Sensing Technology, Sep. 2-4, 2014, Liverpool, UK.

[7] K. Aarthi Priyamvadha, R. Mohanasundaram ,"An Efficient Deduction Of Data Loss Rate In Wireless Sensor Network", in International Journal of Modern Engineering Research (IJMER).

[8] Samiran Gupta, Bappaditya Das, Load Based Reliable Routing In Multi-Sink Sensor Networks, in Research Inventy: International Journal Of Engineering And Science Vol.2,Issue 12 (May 2013), Pp 59-64.

[9] Stefanos A. Nikolidakis, Dionisis Kandris, Dimitrios D. Vergados and Christos Douligeris ,Energy Efficient Routing in Wireless Sensor Networks Through Balanced Clustering, in ISSN 1999-4893, Algorithms 2013.

[10] Meng, Jin-Tao, Jian-Rui Yuan, Sheng-ZhongFeng, and Yan-JieWei. "An Energy efficient Clustering Scheme for Data Aggregation inWireless Sensor Networks." Journal of Computer Science and Technology 28, no. 3 (2013): 564-573.

[11]Deepali Virmani, Ankita Soni, Nikhil Batra,'Reliability Analysis to overcome Black Hole"

\section{Author Profile}

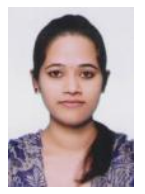

Shraddha Patil received the BE degree in Computer Science from SVERI's College of Engineering Pandharpur and ME degree in Computer Engineering from Jaywantrao Sawant College of Engineering in 2013 and 2017, respectively. During 2013-2017, I have studied the Wireless Sensor Network and recent issues with it, also briefly reviewed intrusion detection in WSN and how to recover from this.

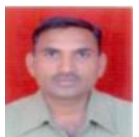

Prof. Madhav Ingle received the MTech degree in Computer Engineering from Dr. Babasaheb Ambedkar Technical University, Lonere, Raigad in 2005 and BE degree in Computer Engineering from Government College Aurangabad in 1997. He is an Assistant Professor with JSPM's Jayawantrao Sawant College of Engineering, Hadapsar, Pune. His research interests span a wide area of networking technologies, distributed system and database. 